ARTICLE OPEN

\title{
Predicting synthesizable multi-functional edge reconstructions in two-dimensional transition metal dichalcogenides
}

\author{
Guoxiang Hu $\mathbb{D}^{1 凶}$, Victor Fung ${ }^{1}$, Xiahan Sang ${ }^{1}$, Raymond R. Unocic (D) ${ }^{1}$ and P. Ganesh $\mathbb{D}^{1 凶}$
}

Two-dimensional (2D) transition metal dichalcogenides (TMDCs) have attracted tremendous interest as functional materials due to their exceptionally diverse and tunable properties, especially in their edges. In addition to the conventional armchair and zigzag edges common to hexagonal 2D materials, more complex edge reconstructions can be realized through careful control over the synthesis conditions. However, the whole family of synthesizable, reconstructed edges remains poorly studied. Here, we develop a computational approach integrating ensemble-generation, force-relaxation, and electronic-structure calculations to systematically and efficiently discover additional reconstructed edges and screen their functional properties. Using MoS $_{2}$ as a model system, we screened hundreds of edge-reconstruction to discover over 160 reconstructed edges to be more stable than the conventional ones. More excitingly, we discovered nine new synthesizable reconstructred edges with record thermodynamic stability, in addition to successfully reproducing three recently synthesized edges. We also find our predicted reconstructed edges to have multi-functional properties-they show near optimal hydrogen evolution activity over the conventional edges, ideal for catalyzing hydrogenevolution reaction (HER) and also exhibit half-metallicity with a broad variation in magnetic moments, making them uniquely suitable for nanospintronic applications. Our work reveals the existence of a wide family of synthesizable, reconstructed edges in 2D TMDCs and opens a new materials-by-design paradigm of 'intrinsic' edge engineering multifunctionality in 2D materials.

npj Computational Materials (2020)6:44; https://doi.org/10.1038/s41524-020-0327-4

\section{INTRODUCTION}

Two-dimensional (2D) transition metal dichalcogenides (TMDCs) have been shown to exhibit exceptional electronic, magnetic, optical, mechanical, and catalytic properties ${ }^{1-5}$. The discontinuity represented by the one-dimensional (1D) edges of 2D TMDCs are analogous to the surfaces of bulk materials, which can impart new properties as well as functionalities ${ }^{6-9}$. For example, 2D $\mathrm{MoS}_{2}$ monolayer is a direct gap semiconductor with a bandgap of $1.8 \mathrm{eV}$, while the 1D $\mathrm{MoS}_{2}$ zigzag (ZZ) edge is metallic ${ }^{10,11}$. Moreover, it is the edges of $2 \mathrm{D}$ TMDCs that are responsible for high catalytic activities, while the basal planes are chemically inert $^{12-15}$. Therefore, understanding the properties as well as the synthesis conditions of the edges in 2D TMDCs is critical to the design of new functional materials.

The lower symmetry of TMDCs compared with graphene, which normally exhibits $Z Z$ or armchair (AC) edges ${ }^{16}$, enables them to present a greater diversity of edge structures and compositions. For example, using molecular beam epitaxy (MBE) under conditions of high Mo flux and atomic-resolution electron microscopy, two types of new reconstructed Mo- terminated edges have been synthesized and imaged. Attributed to the presence of the reconstructed edges, nanoporous $\mathrm{MoS}_{2}$ films were found to have excellent activity for hydrogen evolution reaction (HER) and are magnetic up to $400 \mathrm{~K}^{17}$. Moreover, recent in situ heating experiments using scanning transmission electron microscopy (STEM) to track the edge evolution in monolayer $\mathrm{Mo}_{1-x} \mathrm{~W}_{x} \mathrm{Se}_{2}(x=0.05)$ flakes demonstrated that by varying the local chemical environment, a variety of reconstructed nonstoichiometric edges can be formed ${ }^{18}$. Using first-principles density functional theory (DFT), we have shown that these reconstructed $\mathrm{MoSe}_{2}$ edges can have near optimal HER activity over conventional stoichiometric edges ${ }^{19}$. These studies demonstrated that the edges of TMDCs can be tuned at the nanoscale level, in some cases with high fidelity, giving rise to reconstructions that provide improved functional properties.

Despite these aforementioned successes, the whole family of synthesizable reconstructed edges in 2D TMDCs remains largely unknown. This presents a unique opportunity to computationally screen and discover additional functional reconstructed TMDC edges; in this article, we lay out such a computational approach. Starting with configuration ensemble generations, we screen for stable edges using a computationally affordable force-field method. The obtained stable edges are then further refined with DFT based electronic-structure calculations to generate phase diagrams and screen for their functional properties. Our study thus provides a comprehensive yet affordable computational scheme for predicting synthesizable functional edges of $2 \mathrm{D}$ materials and provides useful guidelines to experimental researchers. Many studies have investigated tuning the catalytic, electronic and magnetic properties of TMDCs by external doping in an Edisonian approach ${ }^{20-23}$, but the merit of our study is to discover a family of 'intrinsically' (based on the metal/chalcogen ratio) tunable materials with widely varying functional properties. Success of our study opens a new materials-by-design paradigm to search and discover other families of 2D 'intrinsic' edge reconstructions with specific multi-functionalities, with potential edge polytypism, for a wide range of nanoscale applications.

\section{RESULTS AND DISCUSSION}

Workflow for predicting synthesizable functional edge reconstructions in 2D TMDCs

We use a nanoribbon model to study the edge reconstructions in 2D TMDCs. As shown in Fig. 1, for a given type $\left(\mathrm{MoS}_{2}, \mathrm{MoSe}_{2}, \mathrm{WS}_{2}\right.$,

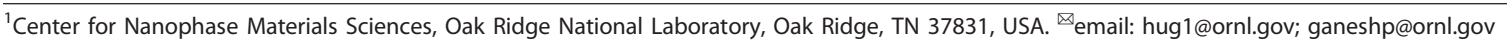



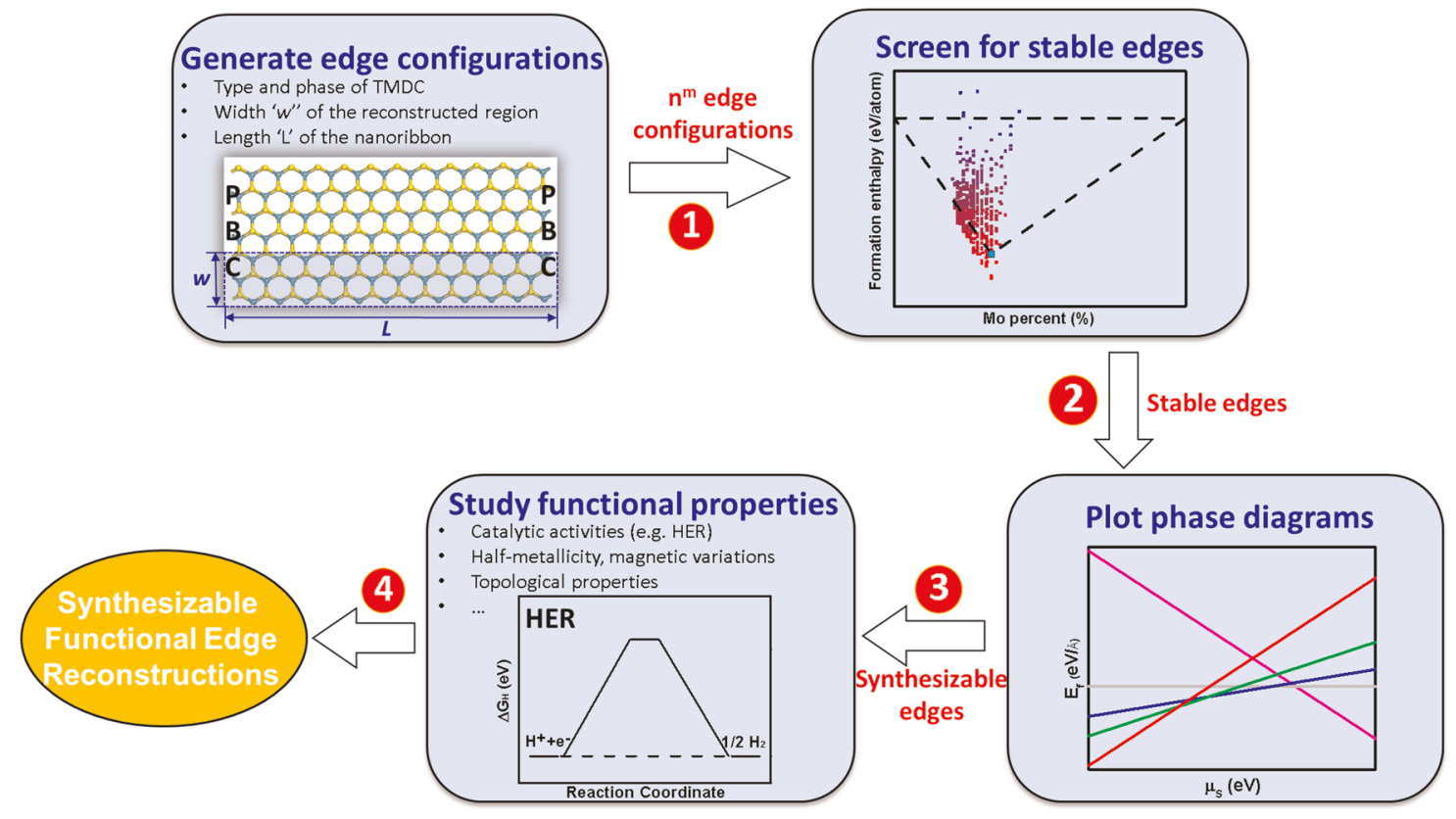

Fig. 1 Workflow for predicting synthesizable multi-functional edge reconstructions in 2D TMDCs. Starting with configuration ensemble generations for a given size of the TMDC nanoribbon in a particular phase, stable edges are screened by using a computationally affordable force field method. The energies of stable edges are then further refined with DFT based electronic-structure calculations to generate phase diagrams to identify synthesizable edge reconstructions, whose functional properties can subsequently be computed.

WSe $\left.e_{2} \ldots\right)$ and phase $\left(2 \mathrm{H}, 1 \mathrm{~T}, 1 \mathrm{~T}^{\prime} \ldots\right)$ of $\mathrm{TMDC}$, we set a region of width ' $w$ ' to look for all possible edge reconstructions in a nanoribbon of length ' $L$ '. The total width of the nanoribbon in our study is seven pairs of Mo and S rows $(\sim 18.96 \AA)$. We then generate $n^{m}$ edge configurations by tiling the underlying triangular lattice, where $m$ and $n$ correspond to the number of rows in the reconstructed region (i.e., $m=w$ ) and number of possible occupancies considered for each row, respectively. For example, in our work with $\mathrm{MoS}_{2}$, we set the width of the reconstructed region to be 4 rows, and consider 5 possible occupancies (blank, Mo, S up, S down, and $\mathrm{S}$ up and down) for each row, to obtain $5^{4}(625)$ edge configurations. After the full set of edge configurations are obtained, we use a cost-effective forcefield method which has been benchmarked and validated against DFT to screen for stable edges. To date, several force-field methods have been developed for TMDCs ${ }^{24-26}$, and here we choose a recently reported reactive force-field (ReaxFF) model for $\mathrm{MoS}_{2}{ }^{27}$. In principle, the structures can be also used to fit a neuralnetwork based surrogate model, as has been shown for other materials ${ }^{28}$. The stable edges are then further refined with explicit DFT calculations to generate phase diagrams and provide guidelines to experimentalists for synthesis. At last, the synthesizable edges are chosen to study the functionalities, including their catalytic activities (e.g., HER), half-metallicity and magnetic variations, with potentially more properties waiting to be discovered, such as topologically protected (magnetic) edgestate for dissipationless quantum nanotransport. In the following sections, we'll apply this workflow to a model system $\mathrm{MoS}_{2}$.

\section{Screening for stable reconstructed edges}

To screen for stable reconstructed edges, we would like to use force fields or surrogate (neural-network-based) methods, as geometry optimizations (and simulated annealing if necessary) of thousands of edges at the DFT level of theory would be computationally prohibitive. Herein we choose a ReaxFF reactive potential, which can adequately describe the thermodynamic and structural properties of $\mathrm{MoS}_{2}$ sheets, guided by extensive DFT simulations ${ }^{27}$. To validate the reliability of ReaxFF in identifying stable edges, we compare the formation energies from ReaxFF with DFT for all the generated 625 edge configurations without geometry optimizations, on both $2 \mathrm{H}$ and $1 \mathrm{~T} \mathrm{MoS}_{2}$ phases. The convex hull plots (formation enthalpy as a function of Mo percent) are shown in Fig. 2a, b, d and e. Both DFT and ReaxFF calculations show that there exists a large quantity of edge configurations which have negative formation enthalpies. Some were found to be even more stable than the conventional unreconstructed edges (as indicated by the cyan dots in the figures), as they fall below the lines that connect the pure elements and the unreconstructed edges. Here, the unreconstructed edge refers to the ZZMo edge. As shown in Fig. $2 \mathrm{c}$ and $\mathrm{f}$, with the exception of data points with unreasonably high formation enthalpies (indicating unstable edges with unphysical atomic geometries), there is a strong linear correlation between the DFT and ReaxFF energies, indicating that the ReaxFF potential can identify stable reconstructed edges.

Next, we use the ReaxFF potential to perform geometry optimizations of the generated 625 edge configurations to screen for stable edges. Figure 3 shows the convex hull plots after geometry optimizations with ReaxFF for $2 \mathrm{H}$ and $1 \mathrm{~T} \mathrm{MoS}_{2}$. One can see that most of the edges become energetically favorable (i.e., have negative formation enthalpies) after geometry optimizations. In addition, $1612 \mathrm{H}$ and $1681 \mathrm{~T} \mathrm{MoS}_{2}$ edges fall below the lines connecting the unreconstructed edges and the pure elements. This indicates a significant portion of reconstructed edges for each phase are more stable than the conventional unreconstructed edges. The left and right slopes of each data point can give an idea about the stability range as a function of chemical potential, but we'll further generate phase diagrams to provide more accurate information on the synthesis conditions where these edges can be experimentally realized.

Screening for synthesizable reconstructed edges

The lowest data points of each stoichiometry shown in Fig. 3 correspond to the edges which are most likely synthesized under specific chemical environments. Thus we choose these edge structures for $2 \mathrm{H} \mathrm{MoS}$ to perform further DFT calculations. The 
(a) $2 \mathrm{H}$ DFT

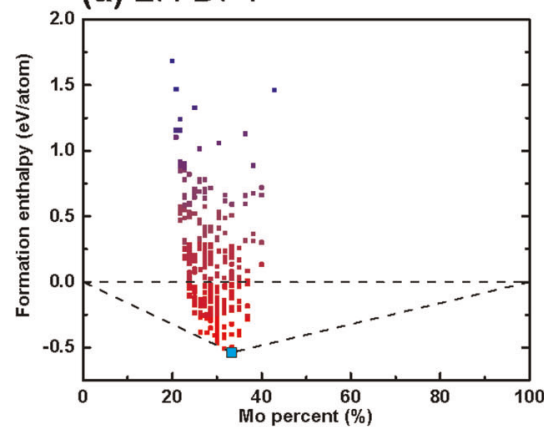

(d) 1 T DFT

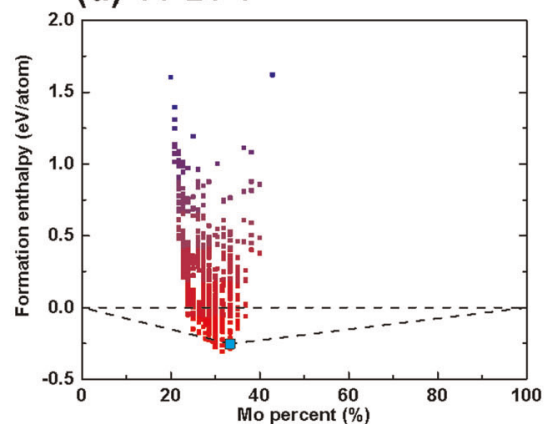

(b) $2 \mathrm{H}$ ReaxFF

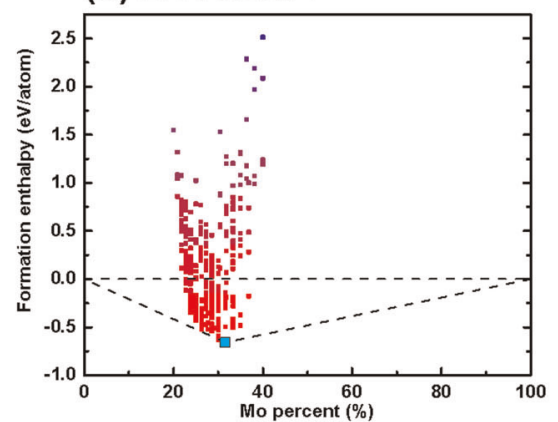

(e) 1T ReaxFF

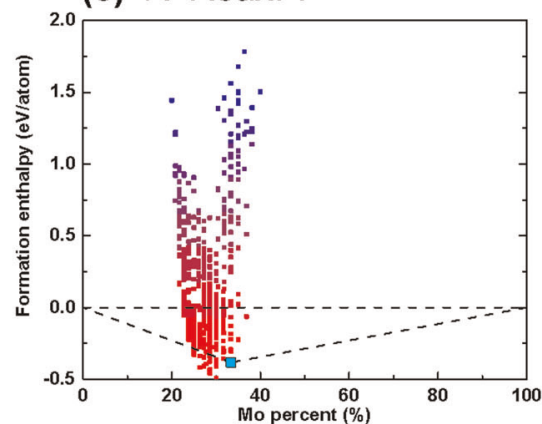

(c) DFT vs. ReaxFF

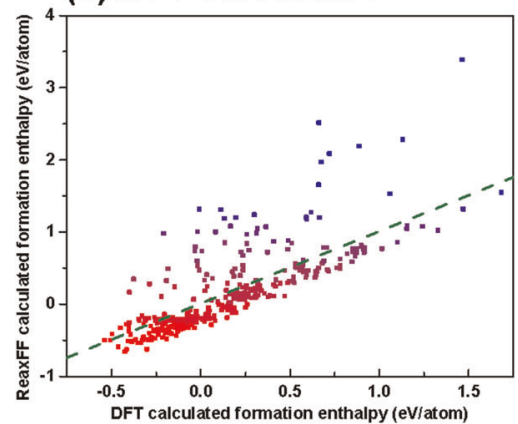

(f) DFT vs. ReaxFF

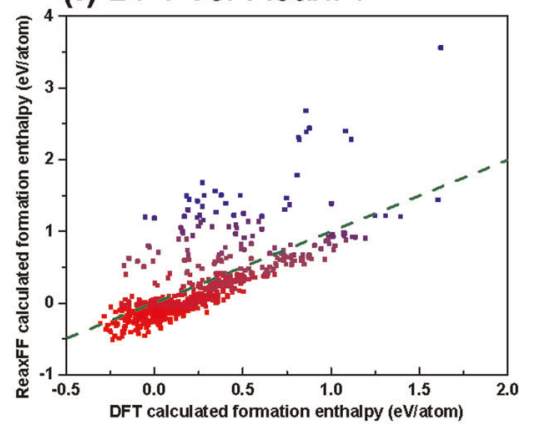

Fig. 2 The convex hull plots without geometry optimizations and the comparison between DFT and ReaxFF energies. $\mathbf{a}$, $\mathbf{b}$, The convex hull plots obtained from DFT and ReaxFF calculations and c, the comparison between DFT and ReaxFF energies for $2 \mathrm{H} \mathrm{MoS} 2 . \mathbf{d}$, e, The convex hull plots obtained from DFT and ReaxFF calculations and $\mathbf{f}$, the comparison between DFT and ReaxFF energies for $1 \mathrm{~T}$ MoS 2 . The cyan dots in (a), (b), (d) and (e) indicate the unreconstructed stoichiometric edges. The green dotted lines in (c) and (d) correspond to $y=x$.

(a) $2 \mathrm{H} \mathrm{MoS}_{2}$

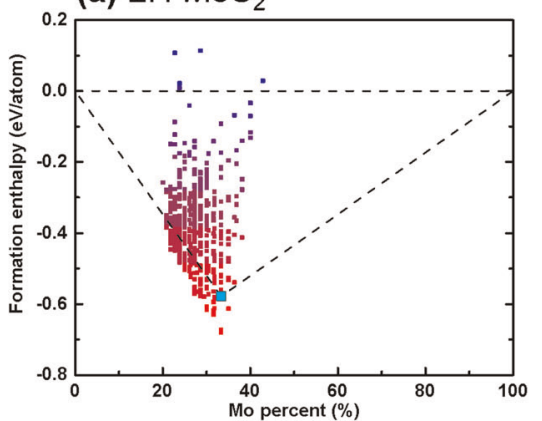

(b) $1 \mathrm{~T} \mathrm{MoS}_{2}$

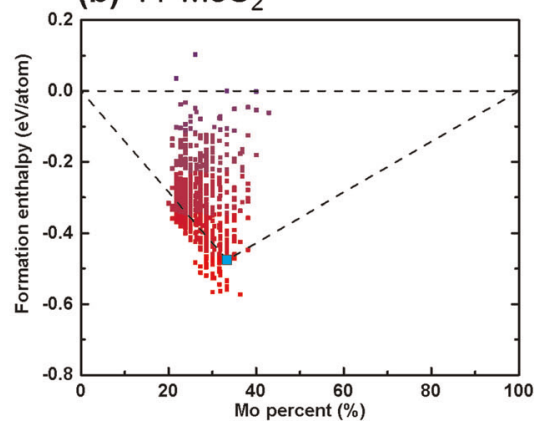

Fig. 3 The convex hull plots after geometry optimizations. a The convex hull plots after geometry optimizations with ReaxFF for $2 \mathrm{H}$ MoS 2 . b The convex hull plots after geometry optimizations with ReaxFF for $1 \mathrm{~T} \mathrm{MoS}$. The cyan dots in (a) and (b) indicate the unreconstructed stoichiometric edges.

reason why we choose the phase of $2 \mathrm{H}$ in this work is that it is more stable than $1 \mathrm{~T}$, and its known edges are active for catalysis $^{12-15}$. Figure 4 shows the DFT refined edge structures for the lowest data points of each stoichiometry. The edges are labeled based on the orientation with respect to the hexagonal lattice of $\mathrm{MoS}_{2}$ (e.g., Mo-oriented ZZMo), and the outermost termination group (e.g., S-terminated (-S) and Mo-terminated (-Mo)). Since our work considers the reconstructions on one side of the nanoribbons, the edges are all derived from the Mooriented unreconstructed edge (ZZMo). We have successfully reproduced 3 Mo-oriented edges found from previous experimental studies: ZZMo, ZZMo-S, and ZZMo-S2 ${ }^{17,18}$, which validates our approach. These edges currently serve as the basis for most studies of physical and chemical properties of $\mathrm{MoS}_{2}$ edges. More excitingly, we have also predicted nine new reconstructed edges with record stability not found in the literature (highlighted in orange). This discovery reveals that there is a large undiscovered set of experimentally realizable edges in the structure space which is being currently overlooked.

Notably, two of the predicted reconstructed edges contain grain boundaries (GBs): ZZMo-GB-Mo and ZZMo-GB-S. GBs in 2D materials connect two regions with different orientations. Various GBs have been observed in $\mathrm{MoS}_{2}$ monolayers, such as the ones made of $4|4,4| 6,4|8,5| 7$, or $6 \mid 8$ ring pairs ${ }^{29-31}$. GBs can affect the properties of $\mathrm{MoS}_{2}$ monolayers significantly. For example, recent study shows that GBs in $\mathrm{MoS}_{2}$ exhibit superior HER catalytic activity, combined with long-term stability ${ }^{32}$. The new predicted ZZMo-GB-Mo edge contains octagon rings, and this type of GB is firstly discovered. Meanwhile, ZZMo-GB-S contains a previously observed $6 \mid 8$ boundary, which switches the orientation of the edge from Mo-oriented to S-oriented.

To better understand the synthesis conditions of the predicted reconstructed edges, we generated the phase diagram with the formation energy as a function of the sulfur chemical potential 


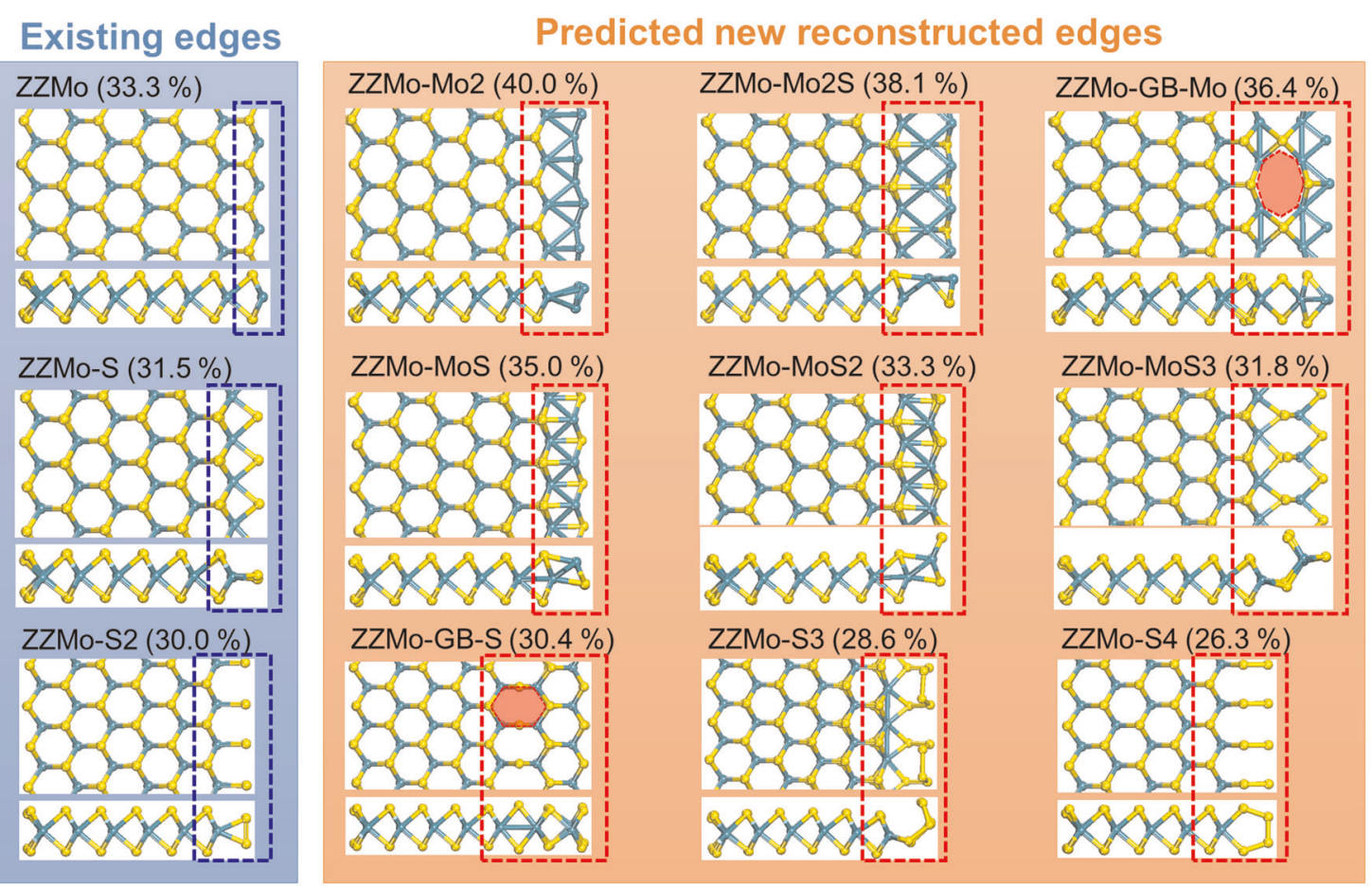

Fig. 4 Top and side views of the predicted stable edges with different stoichiometries. The percentage of Mo in each nanoribbon is shown in bracket. The edges are labeled based on the orientation and the outermost termination group. Mo, cyan; S, yellow.

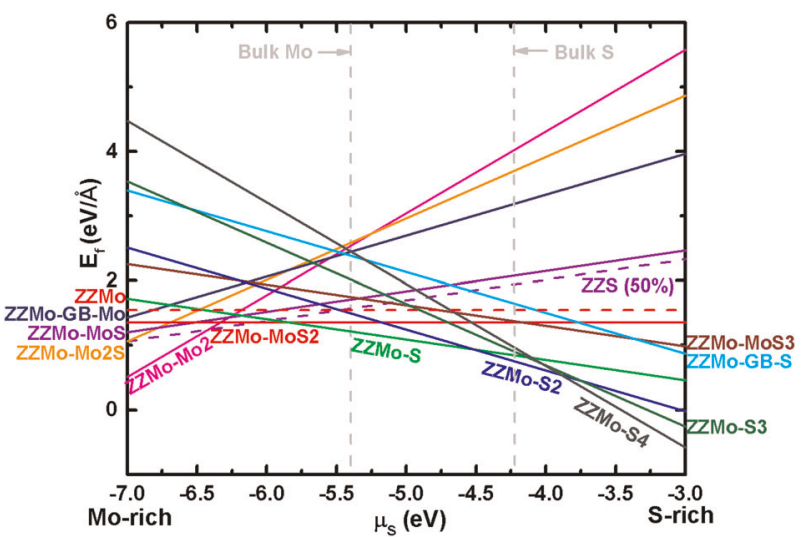

Fig. 5 Formation energies $E_{f}(e V / \AA ̊)$ of the predicted edges as a function of sulfur chemical potential $\mu_{\mathrm{s}}(\mathrm{eV})$. The S-oriented edge with $50 \%$ S coverage ZZS (50\%) was also plotted for comparison. The two vertical dashed lines denote the range $-5.4 \mathrm{eV}<\mu_{\mathrm{s}}<$ $-4.2 \mathrm{eV}$, where $\mathrm{MoS}_{2}$ can remain thermodynamically stable with respect to the formation of bulk Mo $\left(\mu_{\mathrm{S}}=-5.4 \mathrm{eV}\right)$ or bulk ${ }_{\mathrm{S}}\left(\mu_{\mathrm{S}}=\right.$ $-4.2 \mathrm{eV})$.

(Fig. 5). As one can see, the most stable edges from the Mo-rich limit to S-rich limit are ZZMo-Mo2, ZZMo-MoS2, ZZMo-S, ZZMo-S2, and ZZMoS4. Therefore, these edges can be synthesized by tuning the chemical potential via controlled MBE growth ${ }^{17}$ or via controlling heating and electron beam irradiation under STEM ${ }^{18}$. Our results on ZZMo-S and ZZMo-S2 are in agreement with previous studies ${ }^{18}$. Besides the five edges, the other seven edges can also be formed, as they have extremely close formation energies to the five edges under various chemical potential windows. For example, at the sulfur chemical potential of $-3.9 \mathrm{eV}$, the formation energies of ZZMo-S2, ZZMo-S3, and ZZMo-S4 are within $0.1 \mathrm{eV} / \AA$, and could likely coexist. In addition, it is reported that the ZZMo edge (indicated by the red dash line in Fig. 5) can be formed under Mo-rich conditions ${ }^{17}$. ZZMo-Mo2S, ZZMo-MoS, and ZZMo-GB-Mo have lower formation energies than ZZMo under Mo-rich conditions, so it is anticipated that they can also be formed under the same Mo-rich conditions.

For comparison, we have also plotted the often-discussed Soriented edge with $50 \% \mathrm{~S}$ coverage $\mathrm{e}^{33}$ in Fig. 5 . It is a Mo-rich edge with the same stoichiometry as the ZZMo-MoS edge, so it is parallel to ZZMo-MoS in the figure. Compared with the Mo-rich Mo-oriented edges, the S-orientated edge with $50 \% \mathrm{~S}$ coverage is more stable than ZZMo-Mo2S, ZZMo-MoS, ZZMo-GB-Mo, but is less stable than ZZMo-Mo when $\mu_{\mathrm{s}}$ is from -7.0 to $-6.4 \mathrm{eV}$. However, it becomes the most stable edge among all the studied edges in this work when $\mu_{\mathrm{S}}$ increases to the range of -6.4 to $-6.1 \mathrm{eV}$. As $\mu_{\mathrm{S}}$ further increases, the stoichiometric ZZMo-MoS2 becomes the most stable edge, followed by three S-rich Moorientated edges ZZMo-S, ZZMo-S2, and ZZMo-S4.

One point of interest to note is that ZZMo is not the most stable stoichiometric edge (33.3\% Mo). The most stable stoichiometric edge we discovered is the reconstructed ZZMo-MoS2 edge, which is $0.63 \mathrm{eV} /$ supercell lower in energy than the unreconstructed ZZMo edge, and more importantly, $0.35 \mathrm{eV} /$ supercell lower than the currently recognized most stable stoichiometric edge ${ }^{34}$. This energetically strongly preferred stoichiometric ZZMo-MoS2 edge should be considered in future studies of $\mathrm{MoS}_{2}$ edges. It may have interesting consequences in growth. For example, if growth continues from this edge, it could propagate along a different angle, and then a GB which bends the overall sheet structure can be obtained. The metastable edges like ZZMo might be favored due to kinetic stabilization, and they could also be more catalytically active ${ }^{35}$. Thus we select three metastable edges to study to simply demonstrate the viability of the metastable reconstructions. Beyond these, there are hundreds of other metastable edges which are worthy of investigation in the future. Supplementary Figure 1 shows the structures of four metastable edges. ZZMo-S2Mo is another metastable stoichiometric edge, and contains seven-member rings, which often appear in GBs of $\mathrm{MoS}_{2}$ monolayers. ZZMo-S and ZZMo-GB contain 31.5\% Mo (same 
(a)

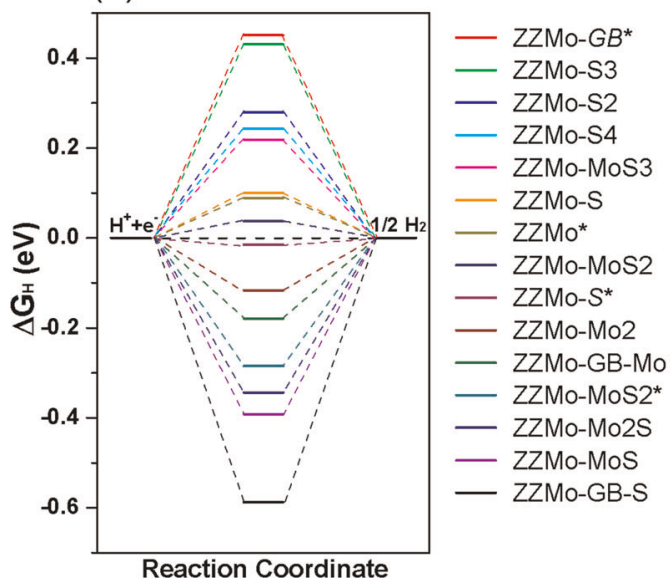

(b)
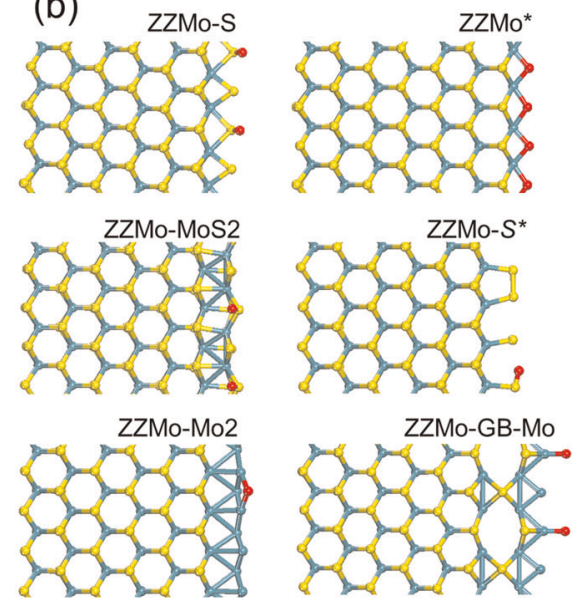

Fig. 6 HER activity on predicted synthesizable reconstructed edges. a Calculated free energy diagram for hydrogen evolution of the predicted synthesizable edges (metastable edges are marked with asterisks). b Structures of $\mathrm{H}$ adsorption at the predicted six most active edges: ZZMo-S, ZZMo, ZZMo-MoS2, ZZMo-S, ZZMo-Mo2, ZZMo-GB-Mo. Mo, cyan; S, yellow; H, red.

stoichiometry as ZZMo-S). ZZMo-S has similar configuration as the observed Mo-Klein edge ${ }^{17}$, while ZZMo-GB contains a $6 / 8$ boundary similar as ZZMo-GB-S, but with less $S$ at the edge. It is expected that these metastable edges can be kinetically stabilized and show promising catalytic activities.

One thing to note is that $\mu_{\mathrm{Mo}}$ and $\mu_{\mathrm{S}}$ need to remain smaller than $\mu_{\mathrm{Mo}}$ (bulk) and $\mu_{\mathrm{s}}$ (bulk), respectively, to avoid the decomposition of $\mathrm{MoS}_{2}$ to bulk Mo or S. Our computed $\mu_{\mathrm{s}}($ bulk $) \approx$ $E_{S}($ bulk $)=-4.2 \mathrm{eV}$, and $\mu_{\text {Mo }}($ bulk $) \approx E_{\text {Mo }}($ bulk $)=-11.5 \mathrm{eV}$. By assuming $\mu_{\mathrm{Mo}}+2 \mu_{\mathrm{S}}=\varepsilon$ at equilibrium, where $\varepsilon$ is the energy per formula unit of $\mathrm{MoS}_{2}$ monolayer, the upper and lower bounds of $\mu_{\mathrm{S}}$ were determined to be -4.2 and $-5.4 \mathrm{eV}$ (as indicated by the two vertical dashed lines in Fig. 5). This is in good agreement with previous studies ${ }^{36}$. Although some of the edges are predicted to be stabilized under conditions beyond this limit, these edges have been observed experimentally due to some kinetic reasons ${ }^{17,18}$.

HER activity on predicted synthesizable reconstructed edges One major promising application for the predicted synthesizable reconstructed edges are for HER, part of the water splitting reaction ${ }^{37-39}$. Electrochemical HER using renewable energy can provide a sustainable supply of fuel for future societies with hydrogen as the key energy carrier ${ }^{40}$. Since $\mathrm{H}$ adsorption is the first step in HER, and the Gibbs free energy for hydrogen adsorption $\left(\Delta G_{H}\right)$ is a well-established descriptor for the HER ${ }^{41-43}$, we screen for HER activity via $\Delta G_{H}$. Figure 6a shows the computed $\Delta G_{H}$ for the fifteen predicted edges (including both stable and metastable edges and the metastable edges are marked with asterisk). Since a close-to-zero value of $\Delta G_{H}$ suggests a good HER catalyst, we predict six edges (ZZMo-S, ZZMo, ZZMo-MoS2, ZZMo$S$, ZZMo-Mo2, and ZZMo-GB-Mo) to be good HER catalysts (the absolute values of $\Delta G_{H}$ for these edges are within $0.2 \mathrm{eV}$ of the optimal point). Our results on the existing edges (ZZMo, ZZMo-S, ZZMo-S2) are in agreement with previous studies ${ }^{14,17}$. Notably, we discovered four new synthesizable reconstructed edges could be better HER catalysts than the existing active edges. Figure $6 \mathrm{~b}$ shows the structures of $\mathrm{H}$ adsorption at the six most active edges. For the S-terminated edges (ZZMo-S, ZZMo-MoS2, and ZZMo-S), H is adsorbed at the $S$ sites, while for the Mo-terminated edges (ZZMo, ZZMo-Mo2, and ZZMo-GB-Mo), $\mathrm{H}$ is adsorbed at the Mo sites. Thus, $\mathrm{H}$ always prefers to adsorb at the under-coordinated sites, as these sites are more active ${ }^{44}$. We have considered $\mathrm{H}$ coverage effects on $\Delta G_{H}$ for all the edges, and the optimal $\mathrm{H}$ coverages (when $\Delta G_{\mathrm{H}}$ is most close-to-zero) of the six most active edges are $50 \%, 100 \%, 50 \%, 25 \%, 25 \%$, and $50 \%$, respectively. $\mathrm{H}$ coverage is defined by $n_{\mathrm{H}} / n_{\text {site, }}$ where $n_{\mathrm{H}}$ is the number of adsorbed $\mathrm{H}$, and $n_{\text {site }}$ is total number of equivalent adsorption site.

Our previous study shows that the optimal HER performance cannot be obtained in the pristine ZZMo and ZZS (or Se) edges ${ }^{19}$. Indeed, here we further prove that the reconstructed edges could achieve better HER performance. Specifically, we find that $\Delta G_{H}$ is $0.10 \mathrm{eV}$ for ZZMo, while it decreased to $0.03 \mathrm{eV}$ for ZZMo-MoS2, and $-0.01 \mathrm{eV}$ for ZZMo-S. Since ZZMo-MoS2 is the most stable stoichiometric edge discovered so far, it is highly expected that this predicted reconstructed edge can be synthesized and applied to HER in the near future. While the energetic difference is not dramatic, a tiny change in $\Delta G_{H}$ near zero could result in a difference of several orders of magnitude improvement in exchange current density ${ }^{45}$. One thing to note is that our calculations assume that the catalyst is charge neutral for computational simplicity, but in reality, the catalyst is usually charged to match its Fermi level with the applied electrode potential. Previous study has shown that due to the charge induced change in the occupation of electronic states, the charge on $2 \mathrm{D}$ materials can have a strong impact on the electrochemical reactions ${ }^{43}$. Therefore, while our current approach looks at the intrinsic HER activities, we hope to further explore HER activities in operating conditions on the reconstructed edges by including the charge effects in the future. Moreover, the chemical interactions between the reaction intermediate adsorbed on the catalyst and the surrounding solvent molecules are neglected in our study. These interactions can be strong for reactions in aqueous solution when the intermediate contains a highly charged atom that can form hydrogen bond(s) with water, for example electrochemical $\mathrm{CO}_{2}$ reduction $\left(\mathrm{CO}_{2} \mathrm{R}\right)^{46}$. Since the adsorbed intermediate $\mathrm{H}$ at the studied edges is nearly charge neutral, it is expected that these interactions won't be very strong, which we also hope to further explore in the future.

\section{Further applications in nanodevices}

Table 1 lists the electronic and magnetic properties of the nanoribbons containing the predicted synthesizable reconstructed edges. It shows that edge reconstructions can effectively modify the properties of the nanoribbons. Specifically speaking, nanoribbons with reconstructed edges could become halfmetallic. Figure 7 shows the density of states (DOS) of the halfmetallic nanoribbon with the unreconstructed ZZS edge on one side and the reconstructed ZZMo-MoS3 edge on the other side. The spin up channel has a gap of $0.38 \mathrm{eV}$, while the spin down channel is metallic. Our spin density and projected DOS plots 
Table 1. Electronic (metallic or half-metallic) and magnetic (magnetic moment $\mu_{\mathrm{B}} /$ supercell) properties of nanoribbons with predicted synthesizable reconstructed edges.

\begin{tabular}{lllllll}
\hline & ZZMo-Mo2 & ZZMo-Mo2S & ZZMo-GB-Mo & ZZMo-MoS & ZZMo-MoS2 & ZZMo \\
\hline Electronic & Metallic & Metallic & Metallic & Metallic & Metallic & Metallic \\
Magnetic & 0.41 & 0.36 & 1.13 & 1.02 & 0.30 & 1.14 \\
\hline & ZZMo-MoS3 & ZZMo-S & ZZMo-GB-S & ZZMo-S2 & ZZMo-S3 \\
\hline Electronic & Half-metallic & Metallic & Metallic & Metallic & Metallic \\
Magnetic & 0.65 & 0.33 & 0.51 & 0.41 & 0.42 & Metallic \\
\hline
\end{tabular}

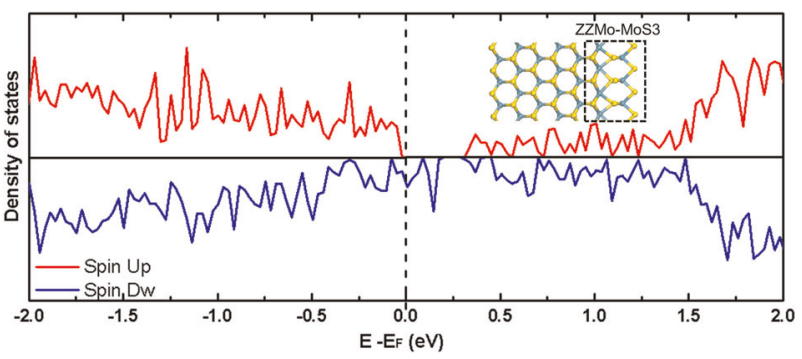

Fig. 7 Density of states (DOS) of the half metallic nanoribbon. The Fermi level (dashed line) is set as zero. The structure of the nanoribbon which has the unreconstructed ZZS edge on one side and the reconstructed ZZMo-MoS3 edge on the other side is also shown.

show that the half-metallicity is mainly from the reconstructed side. The reconstruction-induced peculiar half-metallic nature which allows electrons of only one spin direction to move can be used in spin-based electronics as proposed for half-metallic graphene nanoribbons ${ }^{47,48}$. Meanwhile, the magnetic moments of the nanoribbons vary significantly due to the edge reconstructions. It was recently reported that by integrating multiple magnetic units into a single piece of $\mathrm{MoS}_{2}$ nanoribbon, magnetic devices for information storage can be achieved ${ }^{49}$. Here we provide many synthesizable reconstructed edges with different local magnetic moments, which can further improve the density of information storage.

To further understand potential applications of reconstructed edges in nanodevices, we examine the electronic and magnetic properties of $\mathrm{MoS}_{2}$ nanoribbons with all the generated 625 edge configurations. Interestingly, we find that $412 \mathrm{H} \mathrm{MoS}_{2}$ nanoribbons become half-metallic due to the edge reconstruction, representing a significant proportion of the generated structures. Supplementary Fig. 2 shows the DOS for one of the half-metallic nanoribbons (the geometry is also shown). One can see that near the Fermi level, one spin channel is continuous, while other spin channel has a relatively wide gap of $\sim 0.44 \mathrm{eV}$. Meanwhile for the $1 \mathrm{~T} \mathrm{MoS}_{2}$ nanoribbons, we find they are all metallic, consistent with the fact that $1 \mathrm{~T} \mathrm{MoS}$ monolayer itself is metallic. Regarding their magnetic properties, Fig. 8 shows the histograms of the magnetic moments for the $6252 \mathrm{H}$ and $1 \mathrm{~T} \mathrm{MoS}$ nanoribbons. The pristine $\mathrm{ZZ} \mathrm{MoS}$ nanoribbons possess magnetic moment of $\sim 1.1 \mu_{B} /$ supercell, in agreement with previous studies ${ }^{11}$. However, with edge reconstructions the magnetic moment can be dramatically changed. The range of the magnetic moment for $2 \mathrm{H}$ and $1 \mathrm{~T} \mathrm{MoS}_{2}$ is 0.3 6.2 $\mu_{\mathrm{B}}$ /supercell, and 0.0 4.8 $\mu_{\mathrm{B}}$ /supercell, respectively. Most of the $2 \mathrm{H}$ nanoribbons are in the range of $0.5 \sim 1.5 \mu \mathrm{B} /$ supercell, while most of the $1 \mathrm{~T}$ nanoribbons are in $0.0 \sim 1.0 \mu_{\mathrm{B}}$ /supercell. Therefore, the magnetic moment of a nanoribbon can be increased by 4 6 times by edge reconstructions. These results show that reconstructed edges have great potential applications in nanodevices due to their tunable electronic and magnetic properties.

Given the potential for nanoribbons to host topologically protected states, in the future, it is worthy to investigate how edge reconstructions modify the topology of the electronic-bands and related properties of $\mathrm{MoS}_{2}$ nanoribbons as recently reported for the carbon nanoribbons ${ }^{50}$. It is known that pristine $\mathrm{MoS}_{2}$ nanoribbons demonstrate the quantum valley Hall effect, while some other TMDC nanoribbons present a topological insulator phase $^{51}$. Moreover, 1D topological superconductivity can be realized by placing a TMDC monolayer with $M$ terminated $Z Z$ edge on top of a superconducting substrate ${ }^{52}$. From these revelations one is led to conceive edge reconstructions would affect the topology. Given that topology also plays a role in catalysis $^{53-55}$, there are naturally interesting unexplained implications for (say) HER performance. Applying machine-learning algorithms on our workflow-generated dataset to screen the functional properties of more on-lattice and off-lattice reconstructions, even with larger wavelength reconstructions than we could compute using current DFT-approaches, is another interesting direction.

\section{DISCUSSION}

In conclusion, we have developed a computational approach to rapidly and efficiently discover more synthesizable functional edges in the family of 2D TMDCs. Using $\mathrm{MoS}_{2}$ as an example, we screened 625 edge configurations for $2 \mathrm{H}$ and $1 \mathrm{~T} \mathrm{MoS}$ phases, and predicted stable edges to guide the experimental synthesis. Subsequently we studied the functional properties of these edges. We discover many of these intrinsically tunable edge reconstructions to be near-optimal for HER. More excitingly, they exhibit halfmetallicity and broad variation in local magnetic moments (0.3 6.2 $\mu_{\mathrm{B}} /$ supercell) making them suitable in nanodevices for applications in spintronics and information storage. In the future, the effects of edge reconstructions on the topological properties of TMDC nanoribbons are worthy of further investigations. Combining our current computational approaches to other neural-network and/or evolutionalry structure-search algorithms can lead to accelerating discoveries of more functional edgereconstructions in 2D materials. Our work thus opens a new materials-by-design paradigm, to 'intrinsically' edge engineer multi-functionalities in 2D TMDCs for a wide range of technological applications.

\section{METHODS}

Details of DFT and force field simulations

Spin-polarized DFT calculations were performed using the Vienna ab initio simulation package (VASP) ${ }^{56,57}$. Electron exchange-correlation was represented by the functional of Perdew, Burke and Ernzerhof (PBE) of generalized gradient approximation $(\mathrm{GGA})^{58}$. The ion-electron interaction was described with the projector augmented wave (PAW) method ${ }^{59}$. The plane-wave cutoff is set to $400 \mathrm{eV}$, and a conjugate gradient method is 
(a) $2 \mathrm{H} \mathrm{MoS}$

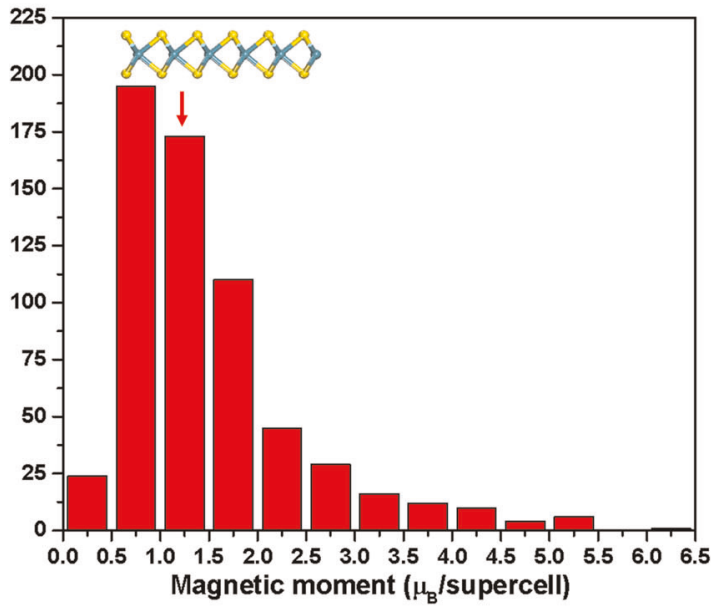

(b) $1 \mathrm{~T} \mathrm{MoS}$

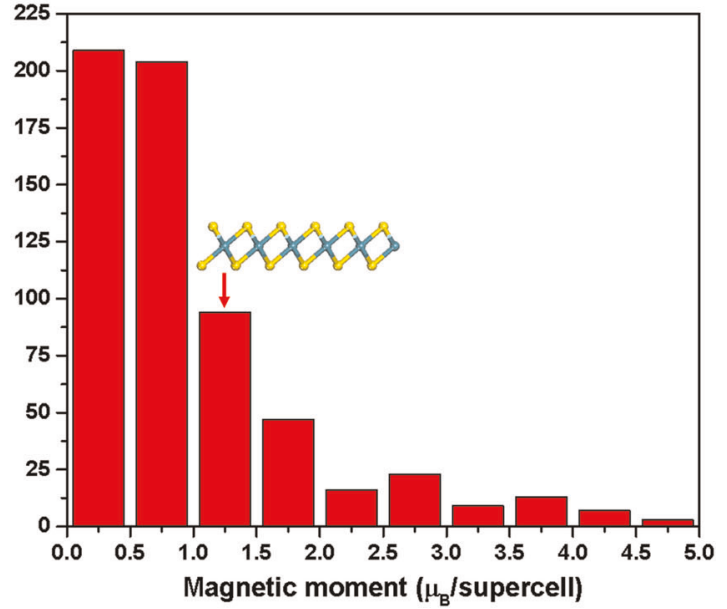

Fig. 8 Histograms of the magnetic moments for the nanoribbons. a Histograms of the magnetic moments for the $2 \mathrm{H}$ nanoribbons with the generated 625 edges. b Histograms of the magnetic moments for the 1T nanoribbons with the generated 625 edges. The structures and magnetic moments of the pristine nanoribbons are also indicated. Mo, cyan; S, yellow.

applied to relax the geometry until interatomic forces are less than $0.025 \mathrm{eV} / \mathrm{A}$. The Brillouin zone was sampled using a $3 \times 1 \times 1$ MonkhorstPack k-point meshes for the $4 \times 1$ supercells. Vacuum spaces in $y$ and $z$ directions are larger than $20 \AA$. . The ReaxFF calculations were performed using Large-scale Atomic/Molecular Massively Parallel Simulator $\left(\right.$ LAMMPS) ${ }^{60}$. The energy in ReaxFF is written as the sum of the bond energy $\left(E_{\text {bond }}\right)$, valence-angle $\left(E_{\text {val }}\right)$, torsion-angle energy $\left(E_{\text {tor }}\right)$, lone pair $\left(E_{\mathrm{Ip}}\right)$, and over-coordinate $\left(E_{\text {over }}\right)$ and under-coordinate $\left(E_{\text {under }}\right)$ energy penalties plus the nonbonded van der Waals $\left(E_{\mathrm{vdw}}\right)$ and Coulomb ( $\left.E_{\text {Coulombic }}\right)$ contributions ${ }^{27}$.

Formation energy calculations

The formation energy per atom was calculated by

$$
E_{f} / A=\frac{E_{\text {tot }}\left(\mathrm{Mo}_{\mathrm{x}} \mathrm{S}_{\mathrm{y}}\right)-x \mu_{\mathrm{Mo}}-y \mu_{\mathrm{s}}}{x+y}
$$

where $E_{\text {tot }}\left(\mathrm{Mo}_{x} \mathrm{~S}_{y}\right)$ is the total energy of the $\mathrm{Mo}_{x} \mathrm{~S}_{y}$ nanoribbon, and $\mu_{\mathrm{Mo}}$ and $\mu_{\mathrm{S}}$ are the chemical potentials of Mo and S. To determine whether the edges can be formed and are stable against decomposition into bulk Mo or bulk S, we constructed convex hull plots in Figs. 2 and 3. The chemical potentials used there are $\mu_{\mathrm{Mo}}$ in body-centered cubic (bcc) Mo and $\mu_{\mathrm{s}}$ in orthorhombic alpha-S, which were obtained from the computed energies per atom at $T=0 \mathrm{~K}$ by neglecting the entropic terms for the condensed states (i.e., $\mu_{\mathrm{s}}($ bulk $) \approx E_{\mathrm{s}}($ bulk $)=-4.2 \mathrm{eV}$, and $\mu_{\mathrm{Mo}_{\mathrm{o}}}(\mathrm{bulk}) \approx E_{\mathrm{Mo}}($ bulk $)=$ $-11.5 \mathrm{eV}$ ). To generate the phase diagram (formation energy as a function of chemical potential), it was assumed that $\mu_{M_{0}}+2 \mu_{\mathrm{S}}=\varepsilon$, where $\varepsilon$ is the energy per formula unit of $\mathrm{MoS}_{2}$ monolayer ${ }^{61}$. Then, the formation energy changed to

$E_{\mathrm{f}}=E_{\text {tot }}\left(\mathrm{Mo}_{\mathrm{x}} \mathrm{S}_{\mathrm{y}}\right)+(2 \mathrm{x}-\mathrm{y}) \mu_{\mathrm{s}}-\mathrm{x} \varepsilon$

$\mathrm{H}$ adsorption energy calculations

The differential $\mathrm{H}$ adsorption energy $\Delta E_{\mathrm{H}}$ was calculated by

$\Delta E_{\mathrm{H}}=E\left(\mathrm{Mo}_{x} \mathrm{~S}_{y}+\mathrm{nH}\right)--E\left(\mathrm{Mo}_{x} \mathrm{~S}_{y}+(n-1) \mathrm{H}\right)-1 / 2 E\left(\mathrm{H}_{2}\right)$

where $E\left(\mathrm{Mo}_{x} \mathrm{~S}_{y}+n \mathrm{H}\right)$ and $E\left[\mathrm{Mo}_{x} \mathrm{~S}_{y}+(n-1) \mathrm{H}\right]$ represent the total energy of the $\mathrm{Mo}_{x} \mathrm{~S}_{y}$ nanoribbon with $n$ and $(n-1)$ adsorbed $\mathrm{H}$ atoms, respectively, and $E\left(\mathrm{H}_{2}\right)$ represents the total energy of a gas phase $\mathrm{H}_{2}$ molecule. $A$ negative value of $\Delta E_{H}$ suggests favorable absorption. The Gibbs free energy of $H$ adsorption $\Delta G_{H}$ was obtained by

$\Delta G_{\mathrm{H}}=\Delta E_{\mathrm{H}}+\Delta E_{\mathrm{ZPE}}-T \Delta \mathrm{S}_{\mathrm{H}}$

where $\Delta E_{\mathrm{ZPE}}$ is the difference in zero-point energy between the adsorbed $\mathrm{H}$ and $\mathrm{H}$ in the gas phase $\mathrm{H}_{2}$ molecule, and $\Delta S_{\mathrm{H}}$ is the entropy difference between the adsorbed $\mathrm{H}$ and $1 / 2 \mathrm{H}_{2}$ in the gas phase under standard conditions. The zero-point energy was calculated by summing vibrational frequencies $\omega$ over normal modes $v: E_{\mathrm{ZPE}}=\frac{1}{2} \sum \hbar \omega$. The entropy of the free $\mathrm{H}_{2}$ molecule at $298.15 \mathrm{~K}$ and $1 \mathrm{~atm}$ was taken from the NIST database.

\section{DATA AVAILABILITY}

Data reported in this paper is available from the corresponding author upon reasonable request.

\section{CODE AVAILABILITY}

Code used to generate the edge configurations in this paper is available from the corresponding author upon reasonable request.

Received: 4 December 2019; Accepted: 10 April 2020; Published online: 05 May 2020

\section{REFERENCES}

1. Wang, Q. H., Kalantar-Zadeh, K., Kis, A., Coleman, J. N. \& Strano, M. S. Electronics and optoelectronics of two-dimensional transition metal dichalcogenides. Nat. Nanotech. 7, 699 (2012)

2. Chhowalla, M. et al. The chemistry of two-dimensional layered transition metal dichalcogenide nanosheets. Nat. Chem. 5, 263 (2013).

3. Manzeli, S., Ovchinnikov, D., Pasquier, D., Yazyev, O. V. \& Kis, A. 2D transition metal dichalcogenides. Nat. Rev. Mater. 2, 17033 (2017).

4. Choi, W. et al. Recent development of two-dimensional transition metal dichalcogenides and their applications. Mater. Today 20, 116-130 (2017).

5. Jariwala, D., Sangwan, V. K., Lauhon, L. J., Marks, T. J. \& Hersam, M. C. Emerging device applications for semiconducting two-dimensional transition metal dichalcogenides. ACS Nano 8, 1102-1120 (2014).

6. Cheng, F. et al. Controlled growth of $1 \mathrm{D} \mathrm{MoSe}_{2}$ nanoribbons with spatially modulated edge states. Nano Lett. 17, 1116-1120 (2017).

7. $\mathrm{Xu}, \mathrm{H}$. et al. Oscillating edge states in one-dimensional $\mathrm{MoS}_{2}$ nanowires. Nat Commun. 7, 12904 (2016).

8. Yin, X. et al. Edge nonlinear optics on a $\mathrm{MoS}_{2}$ atomic monolayer. Science 344 488-490 (2014).

9. Poh, S. M. et al. Large area synthesis of $1 \mathrm{D}-\mathrm{MoSe}_{2}$ using molecular beam epitaxy. Adv. Mater. 29, 1605641 (2017).

10. Bollinger, M. et al. One-dimensional metallic edge states in MoS 2. Phys. Rev. Lett. 87, 196803 (2001).

11. Li, Y., Zhou, Z., Zhang, S. \& Chen, Z. MoS2 nanoribbons: high stability and unusual electronic and magnetic properties. J. Am. Chem. Soc. 130, 16739-16744 (2008).

12. Hinnemann, B. et al. Biomimetic hydrogen evolution: $\mathrm{MoS}_{2}$ nanoparticles as catalyst for hydrogen evolution. J. Am. Chem. Soc. 127, 5308-5309 (2005).

13. Jaramillo, T. F. et al. Identification of active edge sites for electrochemical $\mathrm{H}_{2}$ evolution from $\mathrm{MoS}_{2}$ nanocatalysts. Science 317, 100-102 (2007). 
14. Tsai, C., Chan, K., Abild-Pedersen, F. \& Nørskov, J. K. Active edge sites in $\mathrm{MoSe}_{2}$ and $\mathrm{WSe}_{2}$ catalysts for the hydrogen evolution reaction: a density functional study. Phys. Chem. Chem. Phys. 16, 13156-13164 (2014).

15. Asadi, M. et al. Nanostructured transition metal dichalcogenide electrocatalysts for $\mathrm{CO}_{2}$ reduction in ionic liquid. Science 353, 467-470 (2016).

16. Jia, X., Campos-Delgado, J., Terrones, M., Meunier, V. \& Dresselhaus, M. S. Graphene edges: a review of their fabrication and characterization. Nanoscale $\mathbf{3}$, 86-95 (2011).

17. Zhao, $\mathrm{X}$. et al. Mo-terminated edge reconstructions in nanoporous molybdenum disulfide film. Nano Lett. 18, 482-490 (2017).

18. Sang, X. et al. In situ edge engineering in two-dimensional transition metal dichalcogenides. Nat. Commun. 9, 2051 (2018).

19. Hu, G., Fung, V., Sang, X., Unocic, R. R. \& Ganesh, P. Superior electrocatalytic hydrogen evolution at engineered non-stoichiometric two-dimensional transition metal dichalcogenide edges. J. Mater. Chem. A 7, 18357-18364 (2019).

20. Saab, M. \& Raybaud, P. Tuning the magnetic properties of $\mathrm{MoS}_{2}$ single nanolayers by 3d metals edge doping. J. Phys. Chem. C. 120, 10691-10697 (2016).

21. Park, S. et al. Enhancing catalytic activity of $\mathrm{MoS}_{2}$ basal plane S-vacancy by Co cluster addition. ACS Energy Lett. 3, 2685-2693 (2018).

22. Wang, $\mathrm{H}$. et al. Transition-metal doped edge sites in vertically aligned $\mathrm{MoS}_{2}$ catalysts for enhanced hydrogen evolution. Nano Res. 8, 566-575 (2015).

23. Tedstone, A. A., Lewis, D. J. \& O'Brien, P. Synthesis, properties, and applications of transition metal-doped layered transition metal dichalcogenides. Chem. Mater. 28, 1965-1974 (2016).

24. Wakabayashi, N., Smith, H. \& Nicklow, R. Lattice dynamics of hexagonal MoS studied by neutron scattering. Phys. Rev. B 12, 659 (1975).

25. Jiang, J. W. Parametrization of Stillinger-Weber potential based on valence force field model: application to single-layer MoS2 and black phosphorus. Nanotechnology 26, 315706 (2015).

26. Liang, T., Phillpot, S. R. \& Sinnott, S. B. Parametrization of a reactive many-body potential for Mo-S systems. Phys. Rev. B 79, 245110 (2009).

27. Ostadhossein, A. et al. ReaxFF reactive force-field study of molybdenum disulfide $\left(\mathrm{MoS}_{2}\right)$. J. Phys. Chem. Lett. 8, 631-640 (2017).

28. Behler, J. First principles neural network potentials for reactive simulations of large molecular and condensed systems. Angew. Chem. Int. Ed. 56, 12828-12840 (2017).

29. Zhou, W. et al. Intrinsic structural defects in monolayer molybdenum disulfide. Nano Lett. 13, 2615-2622 (2013).

30. Najmaei, S. et al. Vapour phase growth and grain boundary structure of molybdenum disulphide atomic layers. Nat. Mater. 12, 754 (2013).

31. Van Der Zande, A. M. et al. Grains and grain boundaries in highly crystalline monolayer molybdenum disulphide. Nat. Mater. 12, 554 (2013).

32. Zhu, J. et al. Boundary activated hydrogen evolution reaction on monolayer $\mathrm{MoS}_{2}$. Nat. Commun. 10, 1348 (2019).

33. Schweiger, H., Raybaud, P., Kresse, G. \& Toulhoat, H. Shape and edge sites modifications of $\mathrm{MoS}_{2}$ catalytic nanoparticles induced by working conditions: a theoretical study. J. Catal. 207, 76-87 (2002).

34. Cui, P. et al. Contrasting structural reconstructions, electronic properties, and magnetic orderings along different edges of zigzag transition metal dichalcogenide nanoribbons. Nano Lett. 17, 1097-1101 (2017).

35. Zheng, Y., Jiao, Y., Jaroniec, M. \& Qiao, S. Z. Advancing the electrochemistry of the hydrogen-evolution reaction through combining experiment and theory. Angew. Chem. Int. Ed. 54, 52-65 (2015).

36. Raybaud, P., Hafner, J., Kresse, G., Kasztelan, S. \& Toulhoat, H. Ab initio study of the $\mathrm{H}_{2}-\mathrm{H}_{2} \mathrm{~S} / \mathrm{MoS}_{2}$ gas-solid interface: the nature of the catalytically active sites. J. Catal. 189, 129-146 (2000)

37. Li, Y. et al. $\mathrm{MoS}_{2}$ nanoparticles grown on graphene: an advanced catalyst for the hydrogen evolution reaction. J. Am. Chem. Soc. 133, 7296-7299 (2011).

38. Voiry, D., Yang, J. \& Chhowalla, M. Recent strategies for improving the catalytic activity of 2D TMD nanosheets toward the hydrogen evolution reaction. Adv. Mater. 28, 6197-6206 (2016).

39. Zhang, $X$. et al. Computational screening of $2 D$ materials and rational design

of heterojunctions for water splitting photocatalysts. Small Methods 2, 1700359 (2018).

40. Lubitz, W. \& Tumas, W. Hydrogen: an overview. Chem. Rev. 107, 3900-3903 (2007).

41. Nørskov, J. K. et al. Trends in the exchange current for hydrogen evolution. J. Electrochem. Soc. 152, J23-J26 (2005).

42. Zhang, Q. et al. Trends and descriptors of metal-modified transition metal carbides for hydrogen evolution in alkaline electrolyte. ACS Catal. 9, 2415-2422 (2019).

43. Kim, D., Shi, J. \& Liu, Y. Substantial impact of charge on electrochemical reactions of two-dimensional materials. J. Am. Chem. Soc. 140, 9127-9131 (2018).
44. Hu, G., Wu, Z. \& Jiang, D. E. Stronger-than-Pt hydrogen adsorption in a $A_{22}$ nanocluster for the hydrogen evolution reaction. J. Mater. Chem. A 6, 7532-7537 (2018).

45. Shu, H., Zhou, D., Li, F., Cao, D. \& Chen, X. Defect engineering in $\mathrm{MoSe}_{2}$ for the hydrogen evolution reaction: from point defects to edges. ACS Appl. Mater. Interfaces 9, 42688-42698 (2017).

46. Zhao, X. \& Liu, Y. Unveiling the active structure of single nickel atom catalysis: critical roles of charge capacity and hydrogen bonding. J. Am. Chem. Soc. https:// doi.org/10.1021/jacs.9b13872 (2020).

47. Son, Y. W., Cohen, M. L. \& Louie, S. G. Half-metallic graphene nanoribbons. Nature 444, 347 (2006)

48. Kan, E. J., Li, Z., Yang, J. \& Hou, J. Half-metallicity in edge-modified zigzag graphene nanoribbons. J. Am. Chem. Soc. 130, 4224-4225 (2008).

49. Chen, K. et al. Ferromagnetism of $1 \mathrm{~T}^{\prime}-\mathrm{MoS}_{2}$ nanoribbons stabilized by edge reconstruction and its periodic variation on nanoribbons width. J. Am. Chem. Soc. 140, 16206-16212 (2018).

50. Rizzo, D. J. et al. Topological band engineering of graphene nanoribbons. Nature 560, 204 (2018).

51. Dias, A., Qu, F., Azevedo, D. L. \& Fu, J. Band structure of monolayer transitionmetal dichalcogenides and topological properties of their nanoribbons: nextnearest-neighbor hopping. Phys. Rev. B 98, 075202 (2018).

52. Xu, G., Wang, J., Yan, B. \& Qi, X. L. Topological superconductivity at the edge of transition-metal dichalcogenides. Phys. Rev. B 90, 100505 (2014).

53. Li, L., Zeng, J., Qin, W., Cui, P. \& Zhang, Z. Tuning the hydrogen activation reactivity on topological insulator heterostructures. Nano Energy 58, 40-46 (2019).

54. Li, G. et al. Surface states in bulk single crystal of topological semimetal Co3Sn2S2 toward water oxidation. Sci. Adv. 5, eaaw9867 (2019).

55. Tang, M., Shen, H., Qie, Y., Xie, H. \& Sun, Q. Edge-state-enhanced $\mathrm{CO}_{2}$ electroreduction on topological nodal-line semimetal Cu2Si nanoribbons. J. Phys. Chem. C 123, 2837-2842 (2019).

56. Kresse, G. \& Furthmüller, J. Efficient iterative schemes for ab initio total-energy calculations using a plane-wave basis set. Phys. Rev. B 54, 11169-11186 (1996).

57. Kresse, G. \& Furthmüller, J. Efficiency of ab-initio total energy calculations for metals and semiconductors using a plane-wave basis set. Comput. Mater. Sci. 6, 15-50 (1996).

58. Perdew, J. P., Burke, K. \& Ernzerhof, M. Generalized gradient approximation made simple. Phys. Rev. Lett. 77, 3865 (1996).

59. Blöchl, P. E. Projector augmented-wave method. Phys. Rev. B 50, 17953-17979 (1994).

60. Plimpton, S. Fast parallel algorithms for short-range molecular dynamics. J. Comput. Phys. 117, 1-19 (1995).

61. Bollinger, M., Jacobsen, K. W. \& Nørskov, J. K. Atomic and electronic structure of $\mathrm{MoS}_{2}$ nanoparticles. Phys. Rev. B 67, 085410 (2003).

\section{ACKNOWLEDGEMENTS}

This research was supported by the Laboratory Directed Research and Development Program (LDRD) of Oak Ridge National Laboratory, managed by UT-Battelle, LLC, for the U.S. Department of Energy. A portion of this research was conducted at the Center for Nanophase Materials Sciences, which is a DOE Office of Science User Facility. This research used resources of the National Energy Research Scientific Computing Center, a DOE Office of Science User Facility supported by the Office of Science of the U.S. Department of Energy.

This manuscript has been authored by UT-Battelle, LLC under Contract No. DE-AC0500OR22725 with the U.S. Department of Energy. The United States Government retains and the publisher, by accepting the article for publication, acknowledges that the United States Government retains a non-exclusive, paid-up, irrevocable, worldwide license to publish or reproduce the published form of this manuscript, or allow others to do so, for United States Government purposes. The Department of Energy will provide public access to these results of federally sponsored research in accordance with the DOE Public Access Plan (http://energy.gov/downloads/doepublic-access-plan).

\section{AUTHOR CONTRIBUTIONS}

P.G. conceived the idea, designed the study and wrote the workflows. G.H. performed all calculations and analysis. All authors contributed to interpreting the results. G.H. produced the first draft of the manuscript and all authors contributed towards writing the final draft. 


\section{COMPETING INTERESTS}

The authors declare no competing interests.

\section{ADDITIONAL INFORMATION}

Supplementary information is available for this paper at https://doi.org/10.1038/ s41524-020-0327-4

Correspondence and requests for materials should be addressed to G.H. or P.G.

Reprints and permission information is available at http://www.nature.com/ reprints

Publisher's note Springer Nature remains neutral with regard to jurisdictional claims in published maps and institutional affiliations.
Open Access This article is licensed under a Creative Commons Attribution 4.0 International License, which permits use, sharing, adaptation, distribution and reproduction in any medium or format, as long as you give appropriate credit to the original author(s) and the source, provide a link to the Creative Commons license, and indicate if changes were made. The images or other third party material in this article are included in the article's Creative Commons license, unless indicated otherwise in a credit line to the material. If material is not included in the article's Creative Commons license and your intended use is not permitted by statutory regulation or exceeds the permitted use, you will need to obtain permission directly from the copyright holder. To view a copy of this license, visit http://creativecommons. org/licenses/by/4.0/.

(c) The Author(s) 2020 